\title{
TREATMENT NOTE:
}

\section{A CASE OF INTRACTABLE PRURITIS VULVAE TREATMENT WITH PULSED ULTRASOUND}

\author{
C. A. LIGGINS, M.C.S.P., H.T., Dip.T.P."
}

A 67-year-old married African woman was admitted to hospital on 6 April 1979 after a gynaecological investigation. Her main complaints were vaginal pain and itchiness of the vulva of 12 months duration. Pelvic examination showed atrophy of the labia and vaginal mucosa and a cervical polyp. A diagnosis of chronic vulval dystrophy and pruritis vulvae was made.

While an inpatient she was fully investigated for

* Senior Lecturer, Head of Department of Physiotherapy, University of Durban-Westville.

While an in-patient she was fully investigated for Received 1 August 1981. diabetes; glucose tolerance test suggested diabetes mellitus in a mild form. The polyp was removed under general anaesthetic and a vulval biopsy taken; this proved to be negative. The patient was discharged on 10 April.

She was re-admitted in May 1979 with a marked pruritis vulvae. At the time it was considered that this could have been aggravated by her diabetes so she was put on a diabetic reducing diet. In addition, local application of oestrogen to the vulva was tried, but to no effect. She was then referred to the Skin Clinic and steroid cream was prescribed. There was diminution of 
the itch but it dicl not subside completely. After two weeks she was discharged with instructions to continue using the steroid cream.

The patient was again admitted to hospital on 26 March 1980, still complaining of the itch which had persisted during her time at home. The itch at this time was of such intensiy that it caused her to scratch the vulva until it bled. She was again treated with steroid cream but without success. In the middle of April she was referred to the Physiotherapy department with a request for 'Curapulse therapy' (pulsed short-wave diathermy). The physiotherapist's examination showed a depigmented area involving the labia, vulva, perineum and clitoral area. The patient's main complaint was the intractable itch.

As the Curapulse was not available it was decided to try pulsed ultrasound. The patient was treated on four successive days. The first treatment consisted of pulsed ultrasound, in contact with the pruritic area, using glycerine as a coupling medium. The dose was $30,5 \mathrm{watt} / \mathrm{cm}^{-1}$ for 5 minutes. This resulted in some relief which lasted for 16 hours. The second treatment was given at the same intensity for 6 minutes and relief lasted for 20 hours afterwards. The third and fourth treatments were progressed by one minute in each case. After the third treatment the patient reported complete relief of the itch.

She was discharged from hospial on 19 April 1980 , free of all symptoms and not needing topical application of steroid cream. She was given an open appointment to report back to the Physiotherapy Department should the symptoms recur.

A follow-up home visit one month after discharge revealed that mild pruritis had developed again 3 weeks after the patient's discharge from hospital. Arrangements were made for a longer course of ultrasound to be given but unfortunately the patient's husband refused to let her attend.

\section{DISCUSSION}

After the third treatment with pulsed ultrasound the patient reported complete relief of itch for the first time in two years.

In evaluating the treatment the placebo effect must be considered but can probably be discounted on the evidence that changes of treatment earlier did not produce any significant effect. Local application of oestroigen had no effect and steroid creams only a partial effect; at no time did they relieve symptoms completely.

Grönroos et al. (1979) reported on 25 patients who had various chronic vulval lesions with continuous pruritis, treated by pulsed short-wave therapy. Patients were given $10-15$ single treatments with intervals of two days. The beneficial effect of this therapy was either definite or good in $80 \%$ of all the cases.
The writer has found no reference to the use of pulsed ultrasound in the treatment of chronic pruritis vulvae. The notion to use this modality was based on the fact that insonation with ultrasonic energy results in reduced conduction velocity in nerve. Thus it can be used in its pulsed form to treat painful conditions, particularly those which have reacted adversely to the application of heat (Wadsworth and Chanmugan, 1980). Presumably pain relief is due to reduced conduction velocity in nerves carrying pain impulses. Stimuli which give rise to itch are carried mainly in the slow conducting, unmyelinated $\mathrm{C}$ fibres, and it has been postulated that pain fibres carry the sensation of itch in the sensory spinal nerve to the spinothalamic tract and hence to the thalamus (Lotincz, 1971). The findings of Yamamoto et al. (1981) suggest that clinically, the sensations of itch and pain are closely related. Itch is usually made worse by increased local temperature, however with pulsed ultrasound the treatment is relatively athermic.

Without its thermal influence, the effect of ultrasound is mainly mechanical; it could be speculated that the Gate Control Theory of Melzack and Wall (1965) could help to explain the relief of itch experienced by the patient. Glycerine which was used as the coupling medium is a clear, colourless syrupy liquid used as a moistening agent and a solvent for certain drugs; it is not known to have any anti-pruritic properties.

The troublesome and often distressing condition of pruritis vulvae can be resistant to a wide range of conventional treatments. Alternative approaches using pulsed short-wave diathermy and the more easily applied pulsed ultrasound may prove beneficial. It is intended to study further the effects of pulsed ultrasound in the treatment of chronic pruritis vulvae.

\section{Acknowledgement}

I wish to thank Mrs. L. Gumede, Lecturer in Physiotherapy and the Staff of the King Edward VIII Physiotherapy Department for their assistance with this case.

\section{References}

Grönroos, M. (1979). Treatment of vulval epithelial lesions by pulsed high-frequency therapy. Acta Obstet. Gynecol. Scand. 58, $187-189$.

Melzack, R. and Wall, P. D. (1965). Pain mechanisms: A new theory. Science, 150, 971-979.

Lotincz, A. L. (1971). Neurophysiologic reactions of the skin: Pathophysiology of pruritis. In: Fitzpatrick, $T_{2}$ B., Arndt, K. A., Clark, W. H. et al., eds. Dermatology in general medicine. McGraw-Hill. New York. (p. 212.)

Wadsworth, N. and Chanmugan, A. P. P. (1980). Electrophysical agents in physiotherapy. Science Press. (p. 494.) 